\title{
Accelerated consolidation of soft clays and mine tailings using a desktop centrifuge
}

D. Reid School of Civil and Resource Engineering, The University of Western Australia, Australia; and Golder Associates Pty Ltd, Australia

A.B. Fourie School of Civil and Resource Engineering, The University of Western Australia, Australia

S. Watson Formerly School of Civil and Resource Engineering, The University of Western Australia, Australia

\section{Abstract}

Many mine tailings streams contain appreciable quantities of clay minerals, examples including mineral sands fines, diamond and coal tailings. Tests to determine appropriate consolidation properties for these materials, as well as natural clays, are usually very time-consuming and the availability of equipment (such as a Rowe Cell) can sometimes be a limiting factor. This paper describes the development and testing of a modified desktop centrifuge that should eventually allow the determination of a full suite of consolidation material parameters within two days for soils of very low permeability and stiffness. The results of a series of consolidation tests using the desktop centrifuge are presented. The void ratio-effective stress profile is developed from these tests based on centrifuge theory and direct moisture content measurement. Development of a numerical model of the system to allow estimates of the permeability - effective stress profile of tested slurries is currently underway. Encouraging results have been obtained, indicating the ability of this method to provide reasonable results in the time frame outlined. Suggestions for further developments related to this procedure planned at The University of Western Australia (UWA) are outlined.

\section{$1 \quad$ Introduction}

Laboratory testing to develop estimates of consolidation properties forms a crucial component of the design of most tailings storage facilities. For tailings streams with appreciable quantities of clay minerals, such consolidation testing can be time consuming. In addition, there is a relatively limited pool of available testing devices such as a Rowe Cell. This frequently results in consolidation testing forming part of the critical path for a project, by virtue of the delays in testing. When different slurry densities are under consideration, as is often the case in thickened tailings design, testing of these multiple initial conditions can further impact on project timelines. Often, assumed conservative parameters are utilised in the design studies rather than waiting for test results. Improvements to this state of affairs would be of benefit to the tailings industry generally, and thickened tailings specifically.

Modern centrifuge modelling in geotechnical engineering commenced in the 1930s (Schofield, 1980). The use of centrifuges, and awareness of their capabilities, has increased significantly in the past 40 years. They have been utilised to study a wide range of geotechnical problems, including self-weight consolidation. Today there are at least 100 operating at universities and other research settings worldwide (Muir Wood, 2004). One of the benefits of these devices related to consolidation testing is the accelerated time scales that operate at higher accelerations. This can drastically reduce consolidation time. However, owing to the significant investment required to construct and operate full size centrifuges, they will remain primarily in the research domain for the foreseeable future.

This paper presents the development and testing of a modified desktop-sized centrifuge to allow consolidation tests on slurry samples to be conducted in a much shorter time than conventional testing. A desktop-sized scientific centrifuge was purchased and modified to allow testing of four slurry samples at one time. Piezometers were installed at the base of the slurry columns to allow time dependent behaviour 
to be monitored. Obtaining final moisture contents and comparing with the effective stress induced from centripetal acceleration, allows void ratio-effective stress profiles to be determined.

In typical geotechnical centrifuges, the model size is generally quite small compared to the device radius. This allows the acceleration across a sample to be assumed constant. Various guidelines have been published relevant to the applicability of this assumption (Schofield, 1980; Muir Wood, 2004). However, in the case of the small centrifuge described here, this assumption is not valid. This is actually of benefit to the system under consideration. It allows the consolidated slurry to be exposed to a variable final effective stress profile. Hence, void ratio and permeability profiles across a range of effective stresses can be developed from a single test.

The modified centrifuge was utilised to perform consolidation testing on laboratory standard kaolin. The void ratio-effective stress profile was directly determined based on centrifuge theory and moisture content measurements throughout the depth of the sample. Numerical modelling is underway to allow estimates of the permeability-effective stress profile of the test materials to be made.

\section{Previous related work}

The authors are aware of two published descriptions of the use of desktop sized centrifuges to conduct consolidation testing:

- McDermott and King (1998) performed accelerated consolidation trials of Speswhite Kaolin in a scientific centrifuge of a similar scale to the one outlined in this paper. The material was placed into sample holders for consolidation, and extruded and cut following the test for moisture content measurements. Time dependent behaviour was monitored through use of a strobe light to observe settlement of the material over time. In estimating permeability, they made use of a formula developed by Takada and Mikasa (1986). This expression provided an indication of initial permeability based on the approximately linear initial settling rate. However, modelling of the system to obtain the permeability-effective stress profile during testing was not performed.

- El-Shall et al. (1996) performed centrifugal modelling of a number of phosphatic clays. Their device was much smaller than the one utilised in the current work. In their procedure, the material was consolidated at a number of different speeds, and the settlement measured after each spin. They utilised first-order kinetics to estimate permeability at different void ratios. However, in their work, the time dependent behaviour was based on discrete measurements of the settled depth whenever the centrifuge was stopped.

\section{Experimental procedure}

\section{3. $1 \quad$ Testing system}

The accelerated consolidation testing was conducted on a Clements Orbital 420 scientific centrifuge. This centrifuge was selected based on inspection of the varieties used in the UWA Chemistry department, and their expected applicability to this work. The salient components of the system are shown in Figures 1 and 2 , and a schematic section of the columns is shown in Figure 3.

A number of modifications were made to the system by the UWA Engineering Workshop and Electrical Technicians under direction from the authors, including:

- Installation of digital speed control to provide more accurate assessment of the centrifuge rotations per minute (RPM).

- Replacement of the test tube holders with polycarbonate columns to hold slurry samples, including piezometers within a machined recess at the base. 
- Installation of a transmitter on the top of the centrifuge to transmit readings from the piezometers to a receiver connected to a computer.

Early attempts to perform monitoring of the settlement rate through a strobe light system similar to McDermott and King (1998) were unsuccessful owing to the opacity of the polycarbonate material and smearing of the sidewalls as consolidation occurred. Therefore, monitoring of the time dependent behaviour was conducted using piezometers installed in the base of the chamber.

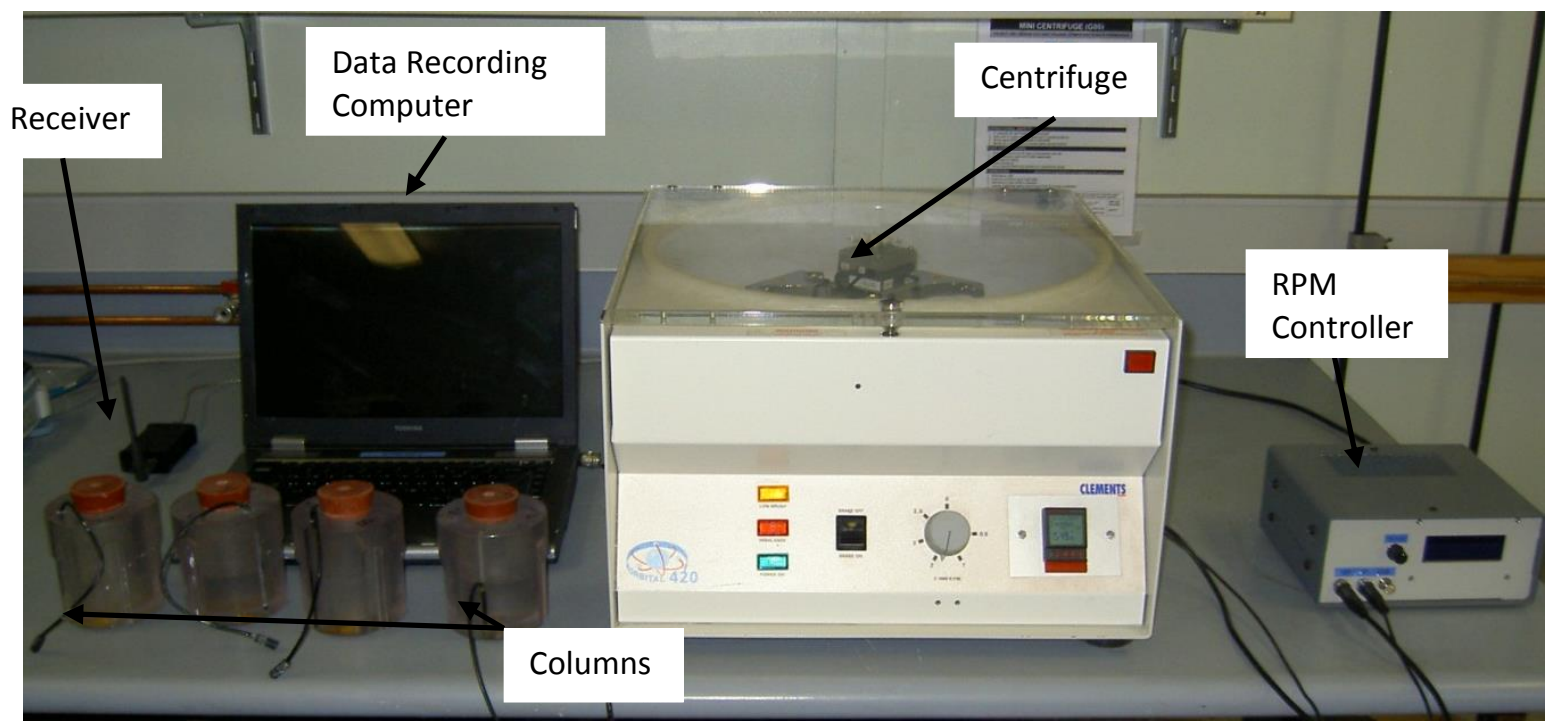

Figure 1 Overall system

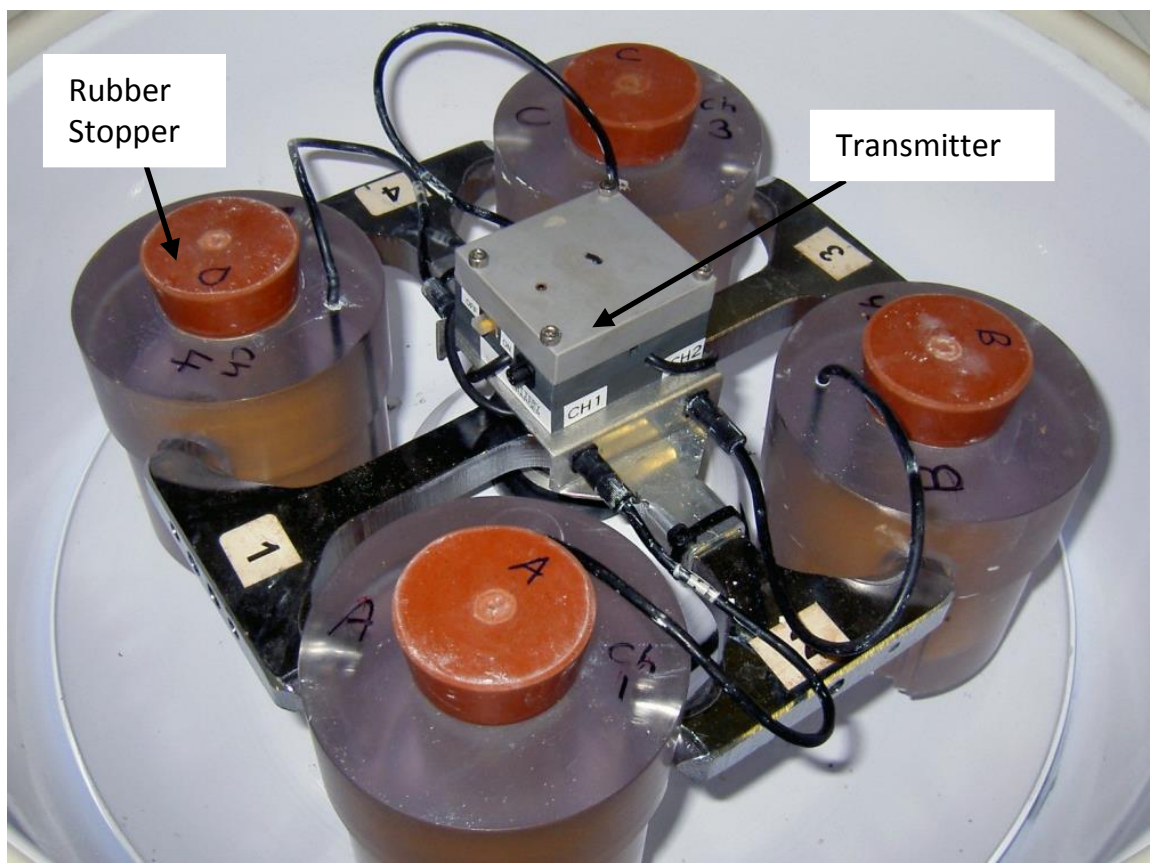

Figure 2 Columns installed for a test 


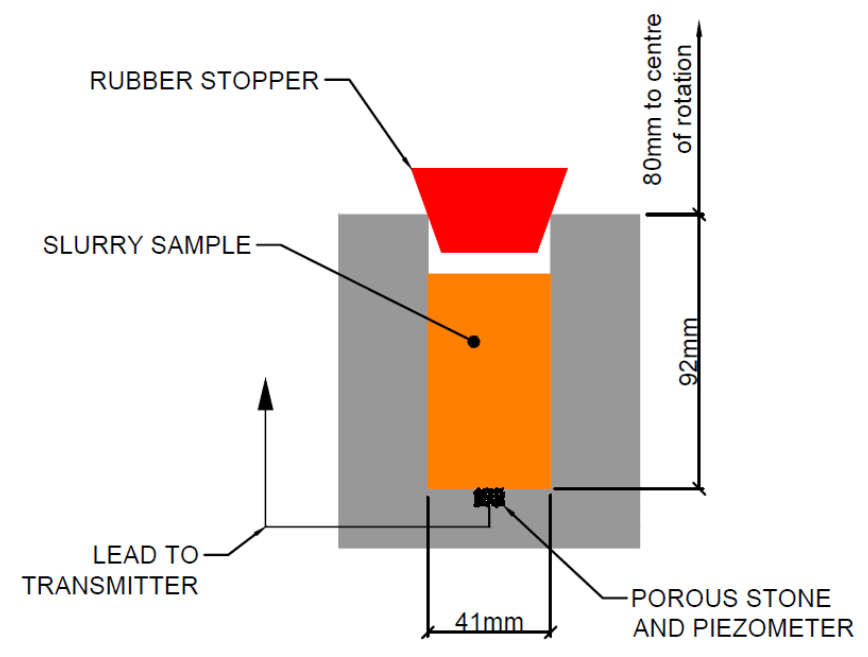

Before Test

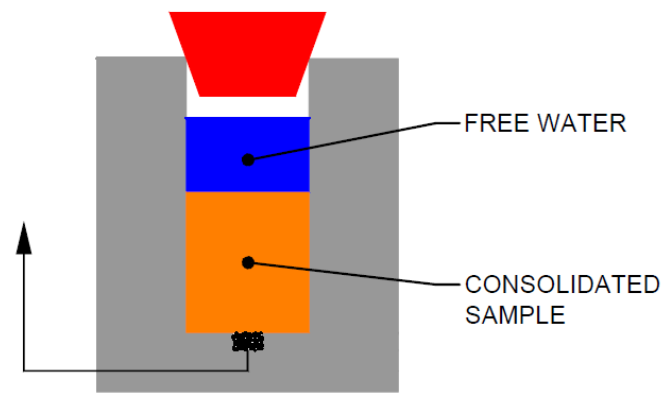

After Test

Figure 3 Schematic column sections

\section{2 Material tested}

The material used for the experimental programme conducted thus far is a kaolin clay, manufactured by Unimin Australia. This material has been used frequently in experimental work at UWA. A comprehensive summary of this material is provided by Lehane et al. (2009). The material has a Liquid Limit of $61 \%$ and a Plasticity Index of $34 \%$. Laser diffraction conducted by the authors indicated this material is $24 \%$ clay sized, with $100 \%$ finer than $75 \mu \mathrm{m}$. The authors measured a specific gravity (SG) of 2.58 for the material.

Kaolin is well suited for early testing and experimental work as there is a significant quantity of available literature on its expected properties. In addition, it can be prepared to a slurry state that is easy to deposit into the testing columns, and has a permeability profile within the range of lower permeability mine slurries. It is also easy to obtain large quantities of relatively consistent material, which aides in testing the repeatability of the system.

\section{3 Sample preparation}

The kaolin was prepared by thoroughly mixing equal parts dry powdered kaolin with deionised water to a moisture content of $100 \%$.

After mixing, the slurry samples were de-aired for at least two hours with a vacuum pump applying a suction of $100 \mathrm{kPa}$. Prior to placement of the slurry into the columns, the columns were half filled with water. A specially manufactured rubber stopper with a pressure line installed was then attached to the columns, as shown in Figure 4. A vacuum suction of $100 \mathrm{kPa}$ was then applied to each column for about 15 minutes to de-air the filter stone recess at the base on the column. This rubber stopper system was also utilised to apply controlled pressures to each column - allowing the piezometers to be calibrated. 


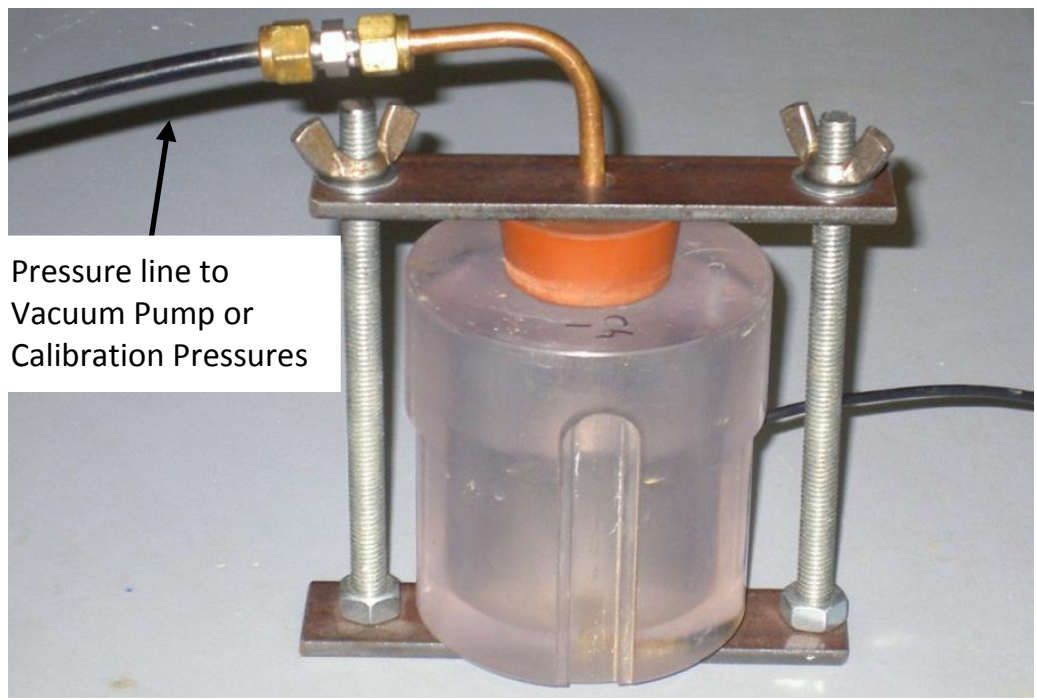

Figure 4 Vacuum application and pressure calibration apparatus

After de-airing was completed, the water in the column was removed except for a thin layer on the base, which left the filter stone recess fully saturated. A sheet of filter paper was then placed over the bottom of the chamber and filter stone. Slurry was then placed in thin layers into the column with a spoon. A glass stirring rod was used to agitate each layer to remove any entrapped air.

Once the sample had been filled to the desired level, each of the chambers were weighed and sample added or removed until the total mass of each of the chambers was within $+/-0.1 \mathrm{~g}$. This was required to ensure the centrifuge was balanced during spinning. Cognisance was taken of the slight differential weights of the rubber stoppers during this process. Upon the weights achieving the required tolerance, the rubber stoppers were inserted, and the chambers placed within the centrifuge.

\section{4 Consolidation procedure}

Following preparation of the samples, spinning of the centrifuge would commence. The system was first gently rotated by hand, to ensure that the chambers were freely hanging from their mounts and could move without interference. The centrifuge lid was then closed, and the rotation of the system increased to a speed of either 1,000 or 1,400 RPM over approximately two minutes. A speed of 1,400 RPM was selected as the maximum for tests conducted thus far, as higher rotations led to vibrations of the system that concerned the authors.

Spinning of the centrifuge continued until the recorded pore water pressures had fallen to a constant level. The drive was then disengaged, and the system slowly braked to a stop.

\subsection{End of test}

Immediately after the system had come to a stop, the chambers were removed, and column plus sample mass obtained. In early runs, it was observed that water was lost through evaporation during spinning. The rate of this loss would not have been precisely known, and would have added unnecessary complication to the numerical modelling to be conducted. In addition, it was conceivable that slightly different rates of evaporation would result in the centrifuge being out of balance. Therefore, the use of rubber stoppers as shown in Figure 2 was implemented. A small hole was drilled into the stoppers to prevent air pressure developing should the rubber stopper be forced further into the column from the centripetal forces. Following commencement of rubber stopper use, no mass loss was observed during testing.

After obtaining sample mass, the rubber stopper was removed and the depths to water surface, and slurry surface were measured. The water was then removed with a syringe. Then, using a specially manufactured 
coring tool, 1 or $2 \mathrm{~mm}$ slices of the material were carefully removed. The slices were placed in individual tins, allowing the moisture content to be obtained. The coring tool utilised is shown in Figure 5.

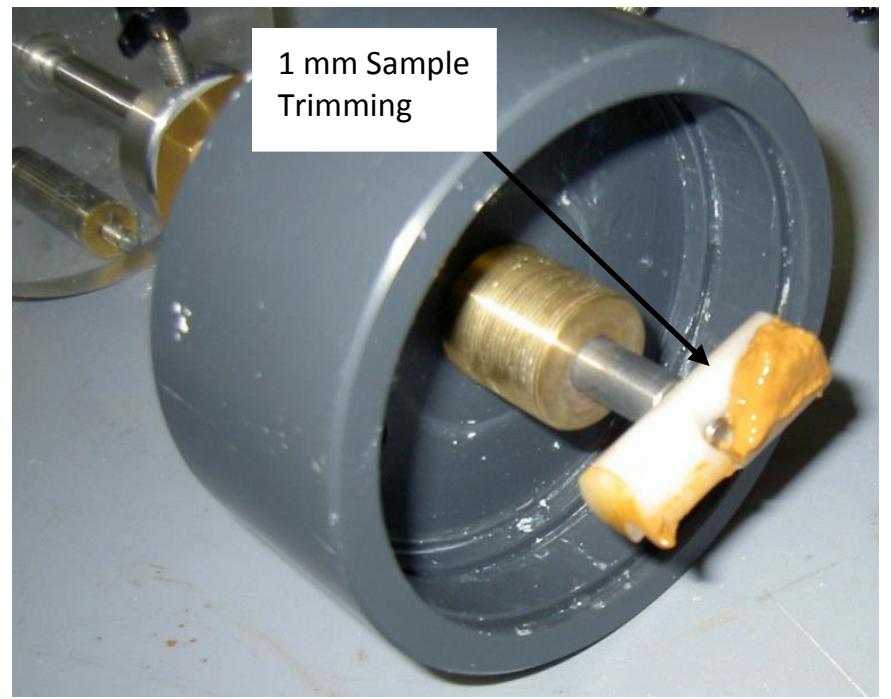

Figure 5 Sample coring tool

\section{$4 \quad$ Results and interpretation}

\section{1 General centrifuge calculations}

Soil within a centrifuge is exposed to a centripetal acceleration equal to $r \omega^{2}$, where $r$ is the radius and $\omega$ is the swept angular velocity. The total stress or equilibrium pore pressure at any radius from the centre of the centrifuge can be calculated incrementally with the following expressions (Muir Wood, 2004):

$$
\begin{aligned}
& \delta \sigma_{t}=\rho_{t} r \omega^{2} \delta z \\
& \delta \mu=\rho_{w} r \omega^{2} \delta z
\end{aligned}
$$

where:

$\delta \sigma_{t} \quad=\quad$ change in total stress across a depth increment.

$\delta \mu \quad=\quad$ change in pore water pressure across a depth increment.

$\rho_{t} \quad=\quad$ bulk density within the depth increment.

$\rho_{w} \quad=\quad$ water density within the depth increment.

$r \omega^{2}=$ centripetal acceleration (sometime normalised to standard gravity, where $N=r \omega^{2} / g$ ).

$\delta z \quad=\quad$ depth increment.

At the commencement of spinning, it can be assumed that the slurry possesses zero effective stress. Hence, total stress and pore water pressure are equal throughout. They were estimated with the above expression based on the bulk slurry density. Calculation of these values at the end of the test requires additional considerations.

\section{2 Void ratio-effective stress}

As outlined above, moisture content measurements were obtained in 1 to $2 \mathrm{~mm}$ increments throughout the height of the samples after consolidation was complete. Assuming saturated condition, and with SG determined separately, this allowed the void ratio at each depth increment to be calculated.

The final effective stress profile was calculated based on the final pore pressure and total stress profile of the sample. The equilibrium pore pressure profile could be easily calculated with the expression outlined 
above, based on the water depth and RPM of the test. Total stress, however, required consideration of the incremental final bulk density profile of the column. This was calculated from the void ratio and water content measurements.

The final profiles of total stress, effective stress, and pore water pressure developed for kaolin at the end of a 1,400 RPM test are shown in Figure 6. The initial total stress profile, based on the as-prepared slurry density, is also shown.

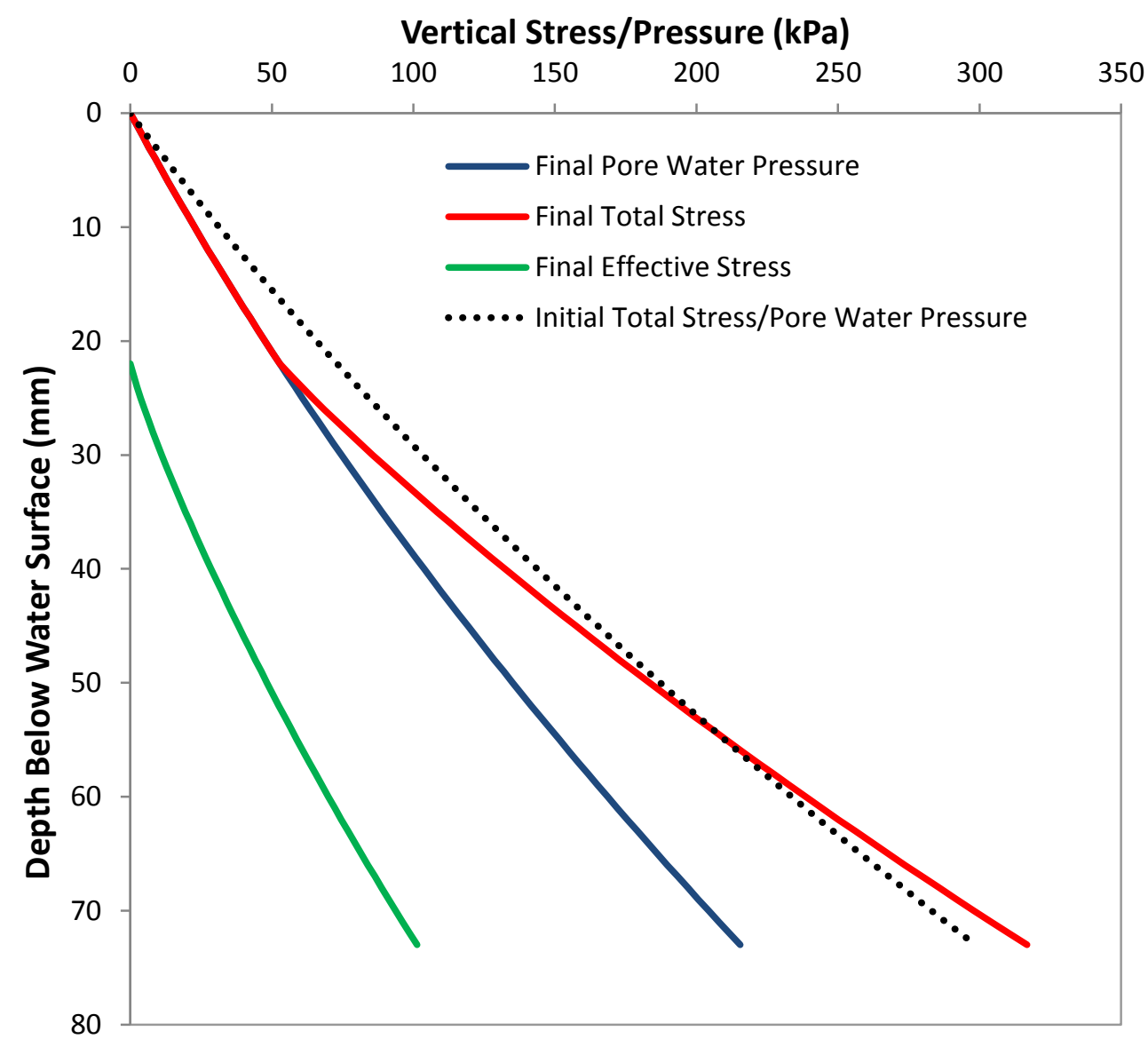

Figure 6 Stress/pressure profile of kaolin sample

The effective stress profile developed above can then be plotted against void ratio determined at various depths, resulting in the results as shown in Figure 7. For comparison, published results from testing on UWA kaolin are shown (Lehane et al. 2009, Stewart 1990). The centrifuge provides good agreement with this data. 


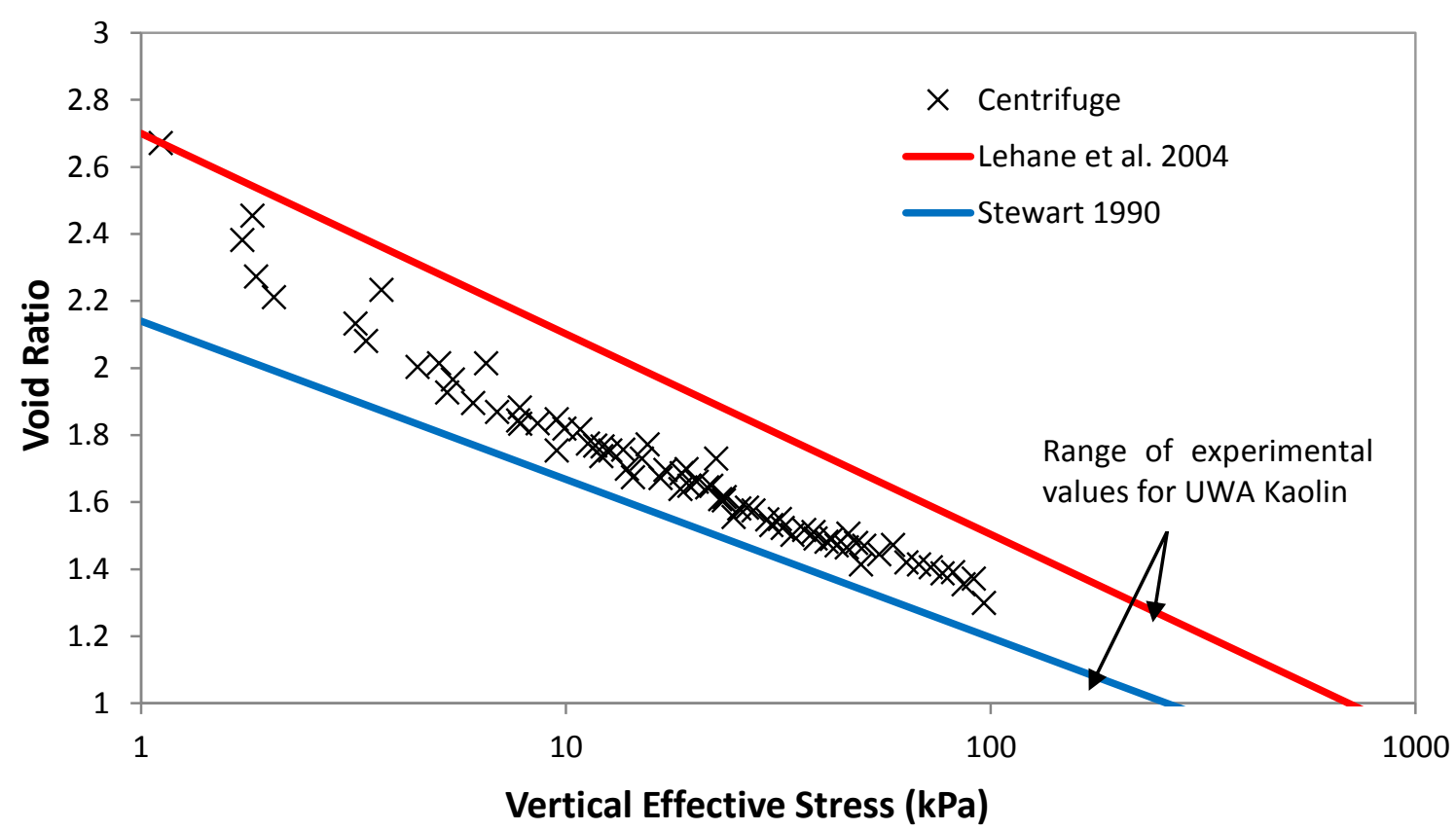

Figure 7 Kaolin - centrifuge void ratio profile compared to previous studies

It should be noted that at the time of writing, two important considerations regarding the interpretation of void ratio have not been explicitly considered:

- Elastic Swelling: as indicated in Figure 6, effective stresses of up to about $100 \mathrm{kPa}$ are being induced on the samples. When the system is brought to a stop, this effective stress will rapidly decrease to a value of essentially zero. This means that elastic swelling likely occurs in the samples prior to sampling. Thus, the samples are in an overconsolidated state. This issue will be addressed further by the authors, with results presented at the conference.

- Creep: owing to the overnight testing often employed, it was rarely the case that the tests would be stopped immediately once excess pore pressure dissipation had ceased. It is therefore likely that some additional creep settlement is occurring in the samples. However, unlike consolidation driven pore water diffusion, which operates under a scale factor of $1 / \mathrm{N}^{2}$ in a centrifuge, creep has a scale factor of 1 (Ko, 1988). It is unlikely that significant creep would occur in the 12-48 hour typical centrifuge run time.

\section{3 Permeability - effective stress interpretation}

Derivation of the full time-dependent behaviour from the settlement or pore pressure response of samples in a centrifuge of the kind described here is not possible using conventional analytical methods. McDermott and King (1998) recognised this, and limited their permeability interpretation to the initial settling rate method of Takada and Mikasa (1986), relevant to the initial void ratio of the sample. This provided excellent estimates of permeability. However, this method suffers from some limitations in the context of typical slurry consolidation testing. The required output of standard consolidation testing is an estimate of the permeability profile across the wide range of effective stress common in a typical tailings facility. For accelerated centrifuge consolidation testing to gain adoption, it will be required to obtain similar parameters. The method that McDermott and King (1998) adapted would make this end impractical to achieve, as it would require a large number of centrifuge runs at different initial void ratios for each sample to be tested. In addition, it would be impractical to prepare samples to the initial void ratio expected at effective pressures beyond which the material behaves as a slurry.

Based on this, it was decided by the authors to develop a numerical model for analysis of the slurry within the centrifuge. In theory, a suitable numerical model should allow different permeability profiles to be 
iteratively trialled in order to match the observed pore pressure decay data. This is currently under development, with promising early results. The status of this work will be presented at the conference.

\section{$5 \quad$ Testing timeframe}

Based on the test work conducted thus far, it appears that the system will result in significantly faster testing times compared to conventional testing. For a kaolin test, a test can be performed, and results obtained in two days. This represents a significant improvement on conventional consolidation testing.

It should be noted that the estimated testing time assumes that only one sample is tested in the four columns. Two samples could be tested simultaneously, while still allowing a duplicate. Also, the time required for the consolidation stage could be decreased through testing at a higher RPM. This will be investigated on future tests, with the results hopefully presented at the conference.

\section{$6 \quad$ Proposed future developments}

While the authors believe the work conducted thus far presents sufficient evidence of the practicality of this device, further work is warranted. Some of this work will likely be conducted as part of the lead author's PhD research, and as a basis for the Honours Theses of a few undergraduate students. It is hoped the results of much of this work will be available for presentation at the conference. Some of the work includes:

- The numerical code under development will be finalised, and validated through modelling the kaolin testing conducted thus. It will then be utilised to provide permeability estimates of other materials, with these results checked through conventional testing.

- Increasing the relevant effective stress range of the device shall be attempted. Increasing the RPM of the machine is one method to achieve this end, however, as this increases both total stress and final pore water pressures, the gains are limited. Therefore, this may be conducted by other means, such as placement of a porous, dense mass onto the sample after initial consolidation is complete, or through decanting the free water and placing additional slurry.

- Limited commercial testing with the device will commence, with the results providing further evidence of the system's utility.

- Development of methods to allow testing of more aggressive $\mathrm{pH}$. The manufacturer of the piezometers currently used cannot give assurances on the range of $\mathrm{pH}$ that the devices can withstand. Hence, only near-neutral samples have been tested. The number of commercially available piezometers that are small enough for installation within this system, and are immune to the effect of high accelerations levels is quite small. Adding the requirement of $\mathrm{pH}$ resistance reduces this pool of available devices much further (to perhaps zero).

- Study of creep effects.

- Study of elastic unloading and the potential requirement to correct for this.

\section{Conclusions}

The development of a modified scientific centrifuge to perform accelerated consolidation testing was outlined. The device was shown to provide reasonable estimates of the void ratio - effective stress profile of kaolin. A numerical model is under development to obtain the permeability profile of the slurry from the test results. The procedure is successful at performing consolidation tests in a much shorter time than conventional testing. Current limitations of the device, and potential research to reduce these, were outlined. 


\section{Acknow l edgements}

The authors would like to acknowledge the substantial advice and specialist skills provided by the UWA Engineering Workshop and Electrical Technicians.

\section{References}

El-Shall, H., Moudgil, B. and Bogan, M. (1996) Centrifugal modelling of the consolidation of solid suspensions, Minerals and Metallurgical Processing SME, Vol. 13, pp. 98-102.

Ko, H.Y. (1988) Summary of the state of the art in centrifuge modelling, in Centrifuges in soil mechanics, W.H. Craing, R.G. James and A.N. Schofield (eds), Taylor and Francis.

Lehane, B.M., O'Loughlin, C.D., Gaudin, C. and Randolph, M.F. (2009) Rate effects on penetrometer in kaolin, Geotechnique ICE, Vol. 59, pp. 41-52.

McDermott, I.R. and King, A.D. (1998) Use of a bench-top centrifuge to assess consolidation, in Proceedings of Tailings and Mine Waste '98 - 5th International Conference, 26-28 January 1998, Fort Collins, Colorado.

Muir Wood, D. (2004) Geotechnical Modelling, E\&FN Spon Press, London, 504 p.

Schofield, A.N. (1980) Cambridge geotechnical centrifuge operations, Geotechnique ICE, Vol. 30, pp. 227-268.

Stewart, D.P. (1990) Lateral loading of piles in soft clay due to nearby embankment construction, Research report GEO: 90086, Department of Civil and Resource Engineering, The University of Western Australia.

Takada, N. and Mikasa, M. (1986) Determination of consolidation parameters by self-weight consolidation test in centrifuge, in Consolidation of Soils: Testing and Evaluation, ASTMSTP 892, R.N. Yong and F.C. Townsend (eds). 